\title{
Zero-range process in random environment
}

\author{
C. Bahadoran, T.S. Mountford, K. Ravishankar and E. Saada
}

\begin{abstract}
We survey our recent articles dealing with one dimensional attractive zero range processes moving under site disorder. We suppose that the underlying random walks are biased to the right and so hyperbolic scaling is expected. Under the conditions of our model the process admits a maximal invariant measure. The initial focus of the project was to find conditions on the initial law to entail convergence in distribution to this maximal distribution, when it has a finite density. Somewhat surprisingly, necessary and sufficient conditions were found. In this part hydrodynamic results were employed chiefly as a tool to show distributional convergence but subsequently we developed a theory for hydrodynamic limits treating profiles possessing densities that did not admit corresponding equilibria. Finally we derived strong local equilibrium results.
\end{abstract}

Key words: Asymmetric attractive zero-range process, site disorder, phase transition, condensation, hydrodynamic limit, strong local equilibrium, large-time convergence

C. Bahadoran

Laboratoire de Mathématiques Blaise Pascal, Université Clermont Auvergne, 63177 Aubière, France. e-mail: Christophe.Bahadoraneuca.fr

T.S. Mountford

Institut de Mathématiques, École Polytechnique Fédérale, Lausanne, Switzerland. e-mail: thomas.mountfordeepfl.ch

K. Ravishankar

NYU-ECNU Institute of Mathematical Sciences at NYU Shanghai, 3663 Zhongshan Road North, Shanghai, 200062, China. e-mail: ravi101048@gmail.com

E. Saada

CNRS, UMR 8145, MAP5, Université de Paris, Campus Saint-Germain-des-Prés, France. e-mail: Ellen.Saada@mi.parisdescartes.fr 


\section{Introduction}

The asymmetric zero-range processs (AZRP) with site disorder was introduced in [19] to study condensation phenomena. It is a conservative interacting particle system whose dynamics is determined by a jump rate function $g: \mathbb{N} \rightarrow \mathbb{N}$, a function $\alpha: \mathbb{Z}^{d} \rightarrow \mathbb{R}_{+}$(called the environment or disorder), and a jump distribution $p($.$) on$ $\mathbb{Z}^{d}$, for $d \geq 1$. A particle leaves site $x$ at rate $\alpha(x) g[\eta(x)]$, where $\eta(x)$ denotes the current number of particles at $x$, and moves to $x+z$, where site $z$ is chosen at random with distribution $p($.$) . As explained later on, this model has a whole family of$ product invariant measures carrying different mean densities; it exhibits a critical density $\rho_{c}$, i.e. no product invariant measure exists above $\rho_{c}([20,18])$, if the function $g$ is bounded, $\alpha$ has averaging properties plus a proper tail assumption. This can be interpreted as a phase transition.

In this review paper, we consider the one-dimensional attractive nearest neighbour process, that is $d=1, p(1)+p(-1)=1$ and $g$ nondecreasing. We summarize the papers [13, 14, 15, 16], by giving their results and the main ideas of their proofs. In these papers, we developed robust approaches to study various aspects of the phase transition mentioned above.

One aspect is the mass escape phenomenon. Suppose the process is started from a given configuration where the global empirical density of particles is greater than $\rho_{c}$. One usually expects convergence to to the extremal invariant measure carrying the same density as the initial state. However, in this case such a measure does not exist. When $g(n)=\min (n, 1)$ and $p(1)=1$ it was shown in [6] that the system converges to the maximal invariant measure (thereby implying a loss of mass). This was established in [13, 14] for the general nearest neighbour model under a weak convexity assumption, and we showed that this could fail for non nearest neighbour jump kernels.

Phase transition also arises in the hydrodynamic limit. We show in [15] that the hydrodynamic behaviour of our process is given under hyperbolic time scaling by entropy solutions of a scalar conservation law

$$
\partial_{t} \rho(t, x)+\partial_{x}[f(\rho(t, x))]=0
$$

where $\rho(t, x)$ is the local particle density field, with a macroscopic flux function $\rho \mapsto f(\rho)$ that is increasing up to $\rho_{c}$ and constant thereafter.

The natural question following hydrodynamic limit is that of local equilibrium. In general, for a conservative particle system endowed with a family $\left(v_{\rho}\right)_{\rho}$ of extremal invariant measuresdenotes the set of allowed macroscopic densities), the local equilibrium property states that the distribution of the microscopic particle configurations at a macroscopic time $t \geq 0$ around a site with macroscopic location $x \in \mathbb{R}$ is close to $v_{\rho(t, x)}$, where $\rho(t, x)$ is the hydrodynamic density, here given by $(1)$. This property has a weak (space-averaged) and a strong (pointwise) formulation, see e.g. [23]. But the local equilibrium property is expected to be wrong at supercritical hydrodynamic densities, that is, such that $\rho(t, x)>\rho_{c}$, since a corresponding equilibrum measure does not exist. This already poses a problem at the level of the 
hydrodynamic limit ([15]), since in the usual heuristic for (17), the macroscopic flux function $f$ is the expectation of microscopic flux function under local equilibrium.

In [16], we introduce a new approach for the derivation of quenched strong local equilibrium. In the case of subcritical hydrodynamic density, that is, $\rho(t, x)<\rho_{c}$, we establish not only conservation but also spontaneous creation of local equilibrium: we only require starting from a sequence of (possibly deterministic) initial configurations with a given macroscopic profile, but with a distribution far away from initial local equilibrium. In the case of supercritical hydrodynamic density $\rho(t, x)>\rho_{c}$, we prove that the local equilibrium property fails, and that, locally around "typical points" of the environment, the distribution of the microscopic state is close to the critical measure denoted by $\mu_{c}^{\alpha}$ : this can be viewed as a dynamic version of the loss of mass property studied in [6, 13, 14]. In the case of a critical hydrodynamic density, that is, $\rho(t, x)=\rho_{c}$, we prove that locally around typical points, the distribution of the system approaches the critical measure. This can still be viewed as a local equilibrium creation result, but only in a partial sense. Indeed, outside the situation of an ergodic disorder (to which we are not limited), the critical measure may not have critical density, nor even any well-defined density.

The paper is organized as follows. In Section 2 we introduce our results through an illustrating analysis of traffic jams. In Section 3, we introduce the model and its basic properties. Section 4 refers to [13, 14], that is, to the convergence to the critical measure $\mu_{c}^{\alpha}$ from a supercritical or a critical initial configuration. Section 5 refers to [15], that is, to the hydrodynamic limits results (including the supercritical regime). Section 6refers to [16], that is, to strong local equilibrium in the subcritical and critical regimes (creation or conservation), and to loss of local equilibrium in the supercritical regime.

\section{A preliminary illustration: traffic jams}

In this section we describe some heuristics for a particular AZRP, where $g(n)=$ $\min (n, 1)$ and $p(1)=1$. This is a well-known model, namely, a series of $M / M / 1$ queues in tandem where each site $x \in \mathbb{Z}$ corresponds to a server with service rate $\alpha(x)$. Then (see e.g. [30]) provided $\lambda<\alpha(x)$ for all $x$, an invariant measure for this process is the product measure whose marginal at site $x$ is the geometric distribution with parameter $1-\lambda / \alpha(x)$ minus 1 , that has mean value $\lambda /(\alpha(x)-\lambda)$. The parameter $\lambda$ is the intensity of the Poisson process of departures from each queue, hence it can be interpreted as the mean current (or flux) of customers along the system. We assume that

$$
\forall x \in \mathbb{Z}, \quad 0<c:=\inf _{y \in \mathbb{Z}} \alpha(y)<\alpha(x)
$$

so that the above invariant measure is defined for $\lambda \leq c$. The value $\lambda=c$ corresponds to what we called "critical" in the introduction. 
This particular AZRP is isomorphic to a totally asymmetric simple exclusion process (TASEP), that models traffic on a one lane highway where overtaking is forbidden. In this TASEP, cars (particles) are labeled from left to right by integers $n \in \mathbb{Z}$, and each of them moves one step further left as long as it is not blocked by another car in front of it (according to the exclusion rule). Particle $n$ jumps with its own rate $\alpha(n)$, herafter called its speed. This intrinsic speed is the one it would reach on an otherwise empty road in the absence of any exclusion rule. Whereas the original AZRP was endowed with site disorder, the associated TASEP is endowed with particle disorder. Here, (2) means that we can find cars moving at speeds arbitrarily close to but not equal to $c$.

The isomorphism between AZRP and TASEP is as follows. An AZRP server at site $n \in \mathbb{Z}$ becomes a TASEP car with label $n$, and customers waiting for this server become vacant TASEP sites between this car and the next one to the left. Thus if we denote by $x_{n}$ the position of the $n$-th TASEP particle, $\eta(n)=x_{n}-x_{n-1}-1$ represents the number of AZRP particles at site $n$.

Any time the $n$-th TASEP particle jumps to the left (that is $x_{n}$ decreases by 1 ), an AZRP customer (i.e. a TASEP hole) is transferred from server $n$ to server $n+1$ at rate $\alpha(n)$ (that is $\eta(n)$ decreases by 1 and $\eta(n+1)$ increases by 1 ). The displacement of car $n$ corresponds to the flux of customers leaving server $n$. By the isomorphism between AZRP and TASEP, invariant measures can be obtained for the above TASEP: under these, inter-particle distances $x_{n+1}-x_{n}$ are independent geometric random variables with parameter $1-\lambda / \alpha(n+1)$, where $0 \leq \lambda \leq c$.

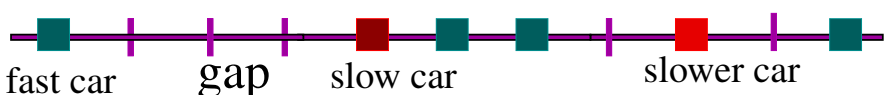

Fig. 1 cars on a highway: fast cars are blue, slow cars are purple, and slower cars are red.

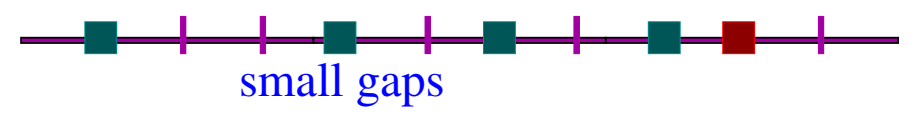

Fig. 2 dense traffic: all cars are separated by small gaps.

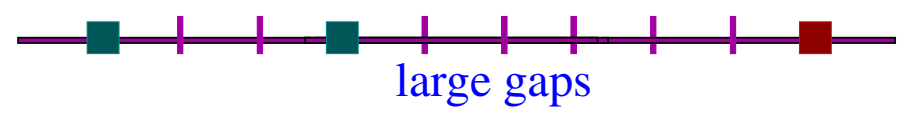

Fig. 3 fluid traffic: all cars are separated by large gaps, but in front of slow cars, the gaps are much larger. 
We illustrate these different cases for TASEP with cars with different speeds (represented by different colors) on a highway, which are now going from left to right (see Fig. 11). When the traffic is dense, cars tend to be slowed down by the exclusion rule, compared to which the differences of speeds between them play a lesser role (see Fig. 2). There is a phase transition between dense and fluid traffic: when the traffic is fluid (see Fig. 3, the difference of speeds plays a dominant role compared to the exclusion rule. There are traffic jams arising behind the slowest cars, and big gaps ahead of them and behind the jams generated by the next slower car (see Fig. 44. Then big gaps get reduced from back to front: the successive jams merge together (see Fig. 5).

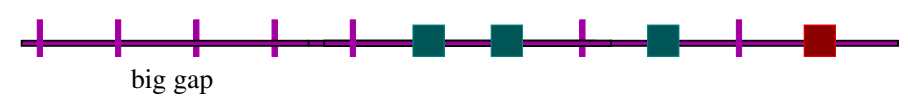

Fig. 4 evolution from fluid traffic

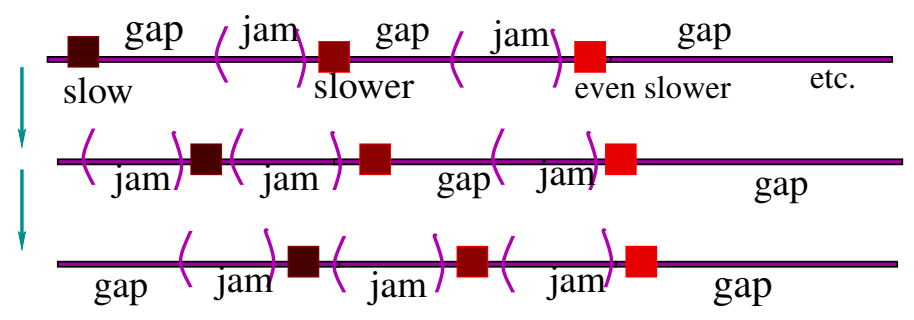

Fig. 5 condensation due to cars regulation: slow cars are dark purple, slower cars are light purple and even slower cars are red

Indeed, to see this, we can define a subsequence $\left\{\alpha_{n_{k}}\right\}$ (of $\left\{\alpha_{n}: n \leq 0\right\}$ ) of rates for successive slower cars which strictly decreases to $c$ such that if $n_{k+1}<j<n_{k}$ then $\alpha_{n_{k}} \leq \alpha_{j}$. This is done by defining $n_{k+1}=\sup \left\{j<n_{k}: \alpha_{j}<\alpha_{n_{k}}\right\}$ and $n_{0}=0$. Let us see how cars with labels in the interval $\mathbb{Z} \cap\left[n_{k+1}, n_{k}\right]$ evolve when we start with large enough gaps. Neglecting the exclusion rule, each car moves at its intrinsic speed as long as it is not slowed down by the motion of the next car ahead. This generates the following scenario:

1. Since car $n_{k}-1$ has greater speed than car $n_{k}$, its displacement will be bigger, hence the gap between them first grows linearly with time, until:

2. in the long run car $n_{k}$ becomes slowed down by the slightly (if $k$ is large) smaller speed of car $n_{k+1}$ and joins the jam already formed behind it by cars with labels inbetween (which are indeed faster then $n_{k+1}$ ). At this time the existing jam behind car $n_{k}$ merges with the one between $n_{k+1}$;

3. on a longer time scale, this new bigger jam will in turn catch up with a slightly slower jam behind car $n_{k+2}$, and so on. 
These steps translate as follows into the AZRP picture, starting with large enough occupation numbers (that is, "supercritical density"):

1. The flux from site $n_{k}-1$ will be greater than the flux leaving site $n_{k}$, and the occupation number of site $n_{k}$ will first grow linearly with time, which can be interpreted as dynamic condensation;

2. in the long run the region between sites $n_{k+1}$ and $n_{k}$ will tend towards an equilibrium with incoming flux close to $\alpha_{n_{k+1}}$ on the left end and an outgoing flux $\alpha_{n_{k}}$ close to matching it, meaning an equilibrium at flux $\alpha_{n_{k+1}}$;

3. when the effect from the next block to the left (i.e. from $n_{k+2}$ to $n_{k}$ ) reaches $n_{k}$, the approximate equilibrium in the site interval $\mathbb{Z} \cap\left[n_{k+2}, n_{k}\right]$ will be one for flux $\alpha_{n_{k+2}}$ on the left.

Since $\alpha_{n_{k}} \rightarrow c$ as $k \rightarrow+\infty$, this suggests that as time goes to infinity the measure on the AZRP will converge to a parametrized by flux $c$. A heuristic derivation of the time scale at which merging (or condensation) occurs, when the service rates $\alpha(x)$ are i.i.d. random variables, can be found in [25].

If we zoom out and look at servers from far away (corresponding to a scaling limit), one obtains a conservation law with a flux function that grows nonlinearly in the "subcritical" density range (that is, for densities with flux less than $c$ ), and is truncated at $c$ in the supercritical range.

While we have described the heuristics for the simple model of totally asymmetric case with a jump rate one if the site is occupied (for AZRP) we obtain the same result for a more complicated case of AZRP (like our general model) where the jumps are asymmetric (but not totally asymmetric), the jump rate is determined by a function depending on the occupation number and the randomness of the disorder is much weaker than independent.

\section{Description of the model, basic properties}

In the sequel, $\mathbb{R}$ denotes the set of real numbers, $\mathbb{Z}$ the set of signed integers, $\mathbb{N}=$ $\{0,1, \ldots\}$ the set of nonnegative integers and $\overline{\mathbb{N}}:=\mathbb{N} \cup\{+\infty\}$. For $x \in \mathbb{R},\lfloor x\rfloor$ denotes the integer part of $x$, that is largest integer $n \in \mathbb{Z}$ such that $n \leq x$. The notation $X \sim \mu$ means that a random variable $X$ has probability distribution $\mu$.

Let $\overline{\mathbf{X}}:=\overline{\mathbb{N}}^{\mathbb{Z}}$ denote the set of particle configurations, and $\mathbf{X}:=\mathbb{N}^{\mathbb{Z}}$ the subset of particle configurations with finitely many particles at each site. A configuration in $\overline{\mathbf{X}}$ is of the form $\eta=(\eta(x): x \in \mathbb{Z})$ where $\eta(x) \in \overline{\mathbb{N}}$ for each $x \in \mathbb{Z}$. The set $\overline{\mathbf{X}}$ is equipped with the coordinatewise order: for $\eta, \xi \in \overline{\mathbf{X}}$, we write $\eta \leq \xi$ if and only if $\eta(x) \leq \xi(x)$ for every $x \in \mathbb{Z}$; in the latter inequality, $\leq$ stands for extension to $\overline{\mathbb{N}}$ of the natural order on $\mathbb{N}$, defined by $n \leq+\infty$ for every $n \in \mathbb{N}$, and $+\infty \leq+\infty$. This order is extended to probability measures on $\overline{\mathbf{X}}$ : For two probability measures $\mu, v$, we write $\mu \leq v$ if and only if $\int f d \mu \leq \int f d v$ for any nondecreasing function $f$ on $\overline{\mathbf{X}}$. We denote by $\left(\tau_{x}\right)_{x \in \mathbb{Z}}$ the group of spatial shifts. For $x \in \mathbb{Z}$, the action of $\tau_{x}$ on 
particle configurations is defined by $\left(\tau_{x} \eta\right)(y)=\eta(x+y)$ for every $\eta \in \overline{\mathbf{X}}, y \in \mathbb{Z}$. Its action on a function $f$ from $\mathbf{X}$ or $\overline{\mathbf{X}}$ to $\mathbb{R}$ is defined by $\tau_{x} f:=f \circ \tau_{x}$.

\subsection{The process and its invariant measures}

Let $p($.$) be a probability measure on \mathbb{Z}$ supported on $\{-1,1\}$. We set $p:=p(1)$, $q:=p(-1)=1-p$, and assume $p \in(1 / 2,1]$, so that the mean drift of the associated random walk is $p-q>0$. Let $g: \mathbb{N} \rightarrow[0,+\infty)$ be a nondecreasing function such that

$$
g(0)=0<g(1) \leq \lim _{n \rightarrow+\infty} g(n)=: g_{\infty}<+\infty .
$$

We extend $g$ to $\overline{\mathbb{N}}$ by setting $g(+\infty)=g_{\infty}$. Without loss of generality, we henceforth assume $g(+\infty)=g_{\infty}=1$.

Let $\alpha=(\alpha(x), x \in \mathbb{Z})$ (called the environment or disorder) be a [0,1]-valued sequence. The set of environments is denoted by $\mathbf{A}:=[0,1]^{\mathbb{Z}}$.

We consider the Markov process $\left(\eta_{t}^{\alpha}\right)_{t \geq 0}$ on $\overline{\mathbf{X}}$ with generator given for any cylinder function (also called local function, that is depending on finitely many sites) $f: \overline{\mathbf{X}} \rightarrow \mathbb{R}$ by

$$
L^{\alpha} f(\eta)=\sum_{x, y \in \mathbb{Z}} \alpha(x) p(y-x) g(\eta(x))\left[f\left(\eta^{x, y}\right)-f(\eta)\right]
$$

where, if $\eta(x)>0, \eta^{x, y}:=\eta-\delta_{x}+\delta_{y}$ denotes the new configuration obtained from $\eta$ after a particle has jumped from $x$ to $y$ (configuration $\delta_{x}$ has one particle at $x$ and no particle elsewhere; addition of configurations is meant coordinatewise). In cases of infinite particle number, the following interpretations hold: $\eta^{x, y}=\eta-\delta_{x}$ if $\eta(x)<\eta(y)=+\infty$ (a particle is removed from $x$ ), $\eta^{x, y}=\eta+\delta_{y}$ if $\eta(x)=+\infty>$ $\eta(y)$ (a particle is created at $y$ ), $\eta^{x, y}=\eta$ if $\eta(x)=\eta(y)=+\infty$.

For the existence and uniqueness of $\left(\eta_{t}^{\alpha}\right)_{t \geq 0}$ see [14, Appendix B]. Recall from [1] that, since $g$ is nondecreasing, $\left(\eta_{t}^{\alpha}\right)_{t \geq 0}$ is attractive, i.e. its semigroup, denoted by $S(t)$ for $t \geq 0$, maps nondecreasing functions (with respect to the partial order on $\overline{\mathbf{X}}$ ) onto nondecreasing functions. A graphical construction via a Harris system ([22]) will be a crucial tool (see e.g. [14]): attractiveness enables to construct a completely monotone coupling of a finite number of copies of the process.

The process $\left(\eta_{t}^{\alpha}\right)_{t \geq 0}$ has the property that if $\eta_{0} \in \mathbf{X}$, then almost surely, one has $\eta_{t} \in \mathbf{X}$ for every $t>0$. In this case, it may be considered as a Markov process on $\mathbf{X}$ with generator (3) restricted to functions $f: \mathbf{X} \rightarrow \mathbb{R}$.

When the environment $\alpha($.$) is identically equal to 1$, we recover the homogeneous zero-range process (see [1] for its detailed analysis).

For $\beta<1$, we define the probability measure $\theta_{\beta}$ on $\mathbb{N}$ by

$$
\theta_{\beta}(n):=Z(\beta)^{-1} \frac{\beta^{n}}{g(n) !}, \quad n \in \mathbb{N}, \quad \text { where } \quad Z(\beta):=\sum_{\ell=0}^{+\infty} \frac{\beta^{\ell}}{g(\ell) !},
$$




$$
g(n) !:=\prod_{k=1}^{n} g(k) \quad \text { for } \quad n \in \mathbb{N} \backslash\{0\}, \quad \text { and } \quad g(0) !:=1 \text {. }
$$

We denote by $\mu_{\beta}^{\alpha}$ the invariant measure of $L^{\alpha}$ defined (see e.g. [18]) as the product measure with marginal $\theta_{\beta / \alpha(x)}$ at site $x$ :

$$
\mu_{\beta}^{\alpha}(d \eta):=\bigotimes_{x \in \mathbb{Z}} \theta_{\beta / \alpha(x)}[d \eta(x)]
$$

Let

$$
c:=\inf _{x \in \mathbb{Z}} \alpha(x)
$$

The measure (4) can be defined on $\overline{\mathbf{X}}$ for $\beta \in[0, c]$, by using the conventions

$$
\begin{aligned}
& \theta_{1}:=\delta_{+\infty}, \\
& \frac{\beta}{a}=0 \text { if } \beta=0 \text { and } a \geq 0 .
\end{aligned}
$$

For $\beta=c, \mu_{c}^{\alpha}$ is by definition the critical measure. The measure (4) is always supported on $\mathbf{X}$ if $\beta \in(0, c) \cup\{0\}$. When $\beta=c>0$, conventions (6)-77) yield a measure supported on configurations with infinitely many particles at all sites $x \in \mathbb{Z}$ that achieve the infimum in (5), and finitely many particles at other sites. In particular, this measure is supported on $\mathbf{X}$ when the infimum in (5) is not achieved. When $c=0$, the measure 4 is supported on the empty configuration.

Since $\left(\theta_{\beta}\right)_{\beta \in[0,1)}$ is an exponential family, we have a stochastic order relation, that is, for $\beta \in\left[0, \inf _{x \in \mathbb{Z}} \alpha(x)\right], \mu_{\beta}^{\alpha}$ is weakly continuous and stochastically increasing with respect to $\beta$.

\subsection{Assumptions on the environment, and consequences}

To state our results, we introduce two sets of assumptions on $\alpha$. The first one says that the environment $\alpha$ has "averaging properties", while the second one sets a restriction on the sparsity of "slow sites" (where by "slow sites" we mean sites where the disorder variable becomes arbitrarily close or equal to the infimum value $c$, which was defined by (5)).

Assumption 1 If $c>0$, there exists a probability measure $Q_{0}=Q_{0}(\alpha)$ on $[0,1]$ such that

$$
Q_{0}(\alpha)=\lim _{n \rightarrow+\infty} \frac{1}{n+1} \sum_{x=-n}^{0} \delta_{\alpha(x)}=\lim _{n \rightarrow+\infty} \frac{1}{n+1} \sum_{x=0}^{n} \delta_{\alpha(x)}
$$

Assumption 2 The environment $\alpha$ has macroscopically dense defects, that is, there exists a sequence of sites $\left(x_{n}\right)_{n \in \mathbb{Z}}$ such that 


$$
\forall n \in \mathbb{Z}, x_{n}<x_{n+1} ; \quad \lim _{n \rightarrow \pm \infty} \alpha\left(x_{n}\right)=c
$$

and

$$
\lim _{n \rightarrow \pm \infty} \frac{x_{n+1}}{x_{n}}=1 .
$$

Remark that 9 implies in particular

$$
\liminf _{x \rightarrow \pm \infty} \alpha(x)=c
$$

By (5) and (8), we have

$$
c=\inf _{x \in \mathbb{Z}} \alpha(x) \leq \inf \operatorname{supp} Q_{0},
$$

and if, for $\beta<1$, we define the mean value of $\theta_{\beta}$ by

$$
R(\beta):=\sum_{n=0}^{+\infty} n \theta_{\beta}(n)
$$

an average mean density exists for all $\beta \in[0, c)$ :

$$
\bar{R}(\beta):=\lim _{n \rightarrow+\infty} \frac{1}{n+1} \sum_{x=-n}^{0} R\left(\frac{\beta}{\alpha(x)}\right)=\lim _{n \rightarrow+\infty} \frac{1}{n+1} \sum_{x=0}^{n} R\left(\frac{\beta}{\alpha(x)}\right) .
$$

Remark 1. When $c>0$, Assumption 1 is actually equivalent to the fact that the two limits in 13] exist and coincide for every $\beta \in[0, c)$.

For reasons of concreteness, we denote by (EE) the stronger hypothesis of environmental ergodicity:

(EE) The environment has a distribution $Q$, for $Q$ a spatially ergodic probability measure on $\mathbf{A}$ with marginal $Q_{0}$, such that $c=\inf \operatorname{supp} Q_{0}$.

For instance, the i.i.d. case $Q=Q_{0}^{\otimes \mathbb{Z}}$ satisfies (EE).

Assumptions 1 and 2 are satisfied under assumption (EE); in particular, $Q$-a.e. realization of the environment $\alpha$ satisfies Assumption 2

By $\left[13, \bar{R}\right.$ is an increasing $C^{\infty}$ function on $[0, c)$ if $c>0$ (see [15, Lemma 3.1]). Define the critical density by

$$
\rho_{c}:=\bar{R}(c-):=\lim _{\beta \uparrow c} \bar{R}(\beta) \in[0,+\infty],
$$

and we define $\bar{R}(c):=\bar{R}(c-)=\rho_{c}$, making $\bar{R}($.$) left continuous at c$. If $c=0, \bar{R}$ is defined on $\{0\}$ and equal to 0 by convention (7). In this case, we set $\rho_{c}=0$.

Moreover, we may define the inverse of $\bar{R}$ on its image $\left[0, \rho_{c}\right)$, and, for $\beta \in[0, c)$, we reindex the invariant measure $\mu_{\beta}^{\alpha}$ by setting 


$$
\mu^{\alpha, \rho}:=\mu_{(\bar{R})^{-1}(\rho)}^{\alpha}
$$

The parameter $\rho$ represents the mean particle density in the sense that, for $\mu^{\alpha, \rho}$ almost every configuration $\eta \in \mathbf{X}$,

$$
\lim _{n \rightarrow+\infty} \frac{1}{n+1} \sum_{x=0}^{n} \eta(x)=\lim _{n \rightarrow+\infty} \frac{1}{n+1} \sum_{x=-n}^{0} \eta(x)=\rho .
$$

Note that under (EE), 10 is satisfied, $\bar{R}(\beta)$ is well-defined by the ergodic theorem for all $\beta \in[0, c]$, and we have

$$
\bar{R}(\beta)=\int_{(c, 1]} R\left[\frac{\beta}{a}\right] d Q_{0}[a], \quad \rho_{c}=\bar{R}(c),
$$

Remark 2. (i) Unlike under (EE), under 8 , $\lim _{\beta \uparrow c} \bar{R}(\beta)$ could be distinct from $\bar{R}(c)$ as defined by 13 for $\beta=c$. In fact, the limits in 13 may not exist for $\beta=c$, or exist and be different from one another and/or from $\rho_{c}$.

(ii) In view of (14), it may be tempting to extend the parametrization (15) to $\rho=\rho_{c}$ by setting $\mu^{\alpha, \rho_{c}}=\mu_{c}^{\alpha}$. However, this does not make real sense, as under (8), because of $(i)$ above, the measure thus defined may not satisfy $\left[16\right.$ for $\rho=\rho_{c}$.

We regard densities below $\rho_{c}$ as subcritical, density $\rho_{c}$ as critical and densities above $\rho_{c}$ as supercritical. In the former there exists an equilibrium probability measure with this density, while we will see that none exists for supercritical densities.

\section{Convergence}

A natural question for an interacting particle system with multiple equilibria is, given a particular equilibrium $\mu$, to determine the set of initial configurations for which the particle system converges to $\mu$. Among classical conservative systems this is fully answered for symmetric exclusion processes on $\mathbb{Z}^{d}$ (see e.g. [28]) but in general seems very hard. It is often softened to finding large classes of initial distributions for which there is convergence. For asymmetric nearest neighbour exclusion processes, the first result of importance was [2], see also [29] and [12] which gave conditions for convergence to product measure. The last is important since it does not require an initial translation invariant distribution but only needs a fixed initial distribution satisfying the appropriate law of averages.

Theorems 1 and 2 below, proved respectively in [13] and [14], establish a necessary and sufficient condition for our zero range process to converge to $\mu_{c}^{\alpha}$. To our knowledge there are no other comparable results for nontrivial assymmetric conservative systems. Certainly the fact (to be justified below), that we consider the maximal equilibrium helps but it is to be noted that, [12] notwithstanding, for the 
exclusion process it is not clear what a necessary and sufficient condition for convergence in distribution to $\delta_{\underline{\underline{0}}}$ (and so $\delta_{\underline{1}}$ ) would be (where $\underline{0}$ and $\underline{1}$ denote the empty and full configurations, defined respectively by $\underline{0}(x)=1-\underline{1}(x)=0$ for every $x \in \mathbb{Z})$.

\subsection{Previous results for convergence}

The main paper we refer to is [6], which considers a TAZRP $(p(1)=1)$, on $\mathbb{Z}$, for $g(n)=\mathbf{1}_{\{n \geq 1\}}$, with $\alpha(x) \in[c, 1]$. Their results are the following:

(a) [6, Theorem 2.1] For $\alpha$ fixed, the geometric product measures $\mu_{\beta}^{\alpha}$, for $\beta<\alpha(x)$ for all $x \in \mathbb{Z}$, are the extremal invariant measures. The range of the parameter $\beta$ may be either $[0, c)$ (when $\alpha(x)=c$ for some $x$ ) or $[0, c]$ (when $\alpha(x)>c$ for all $x)$.

(b) [6, Proposition 2.2] There does not exist any invariant measure with a density above $\rho_{c}$.

(c) [6, Theorem 2.3] If $\rho_{c}<+\infty$, if $\eta_{0} \in \mathbb{N}^{\mathbb{Z}}$ satisfies the supercriticality assumption

$$
\liminf _{n \rightarrow \infty} n^{-1} \sum_{x=-n}^{0} \eta_{0}(x)>\rho_{c},
$$

then $\eta_{t}^{\alpha}$ converges in distribution to $\mu_{c}^{\alpha}$.

Note that under our assumptions, we obtain $\beta \in[0, c]$ in $(a)$.

An immediate consequence of $(c)$ is that there is a "loss of mass": for "most" of the sites the local density will be $\rho_{c}$ but our system is conservative and the hypothesis (18) has a "global" density (at least on the left halfline) strictly higher.

This is an example of condensation (as exhibited for instance in [19, 25, 20]). The "lost" mass accumulates at sites with $\alpha$ value close to the minimum $c$ (where "close" decreases with time). If we return to $M / M / 1$ queues with service rate $\alpha(x)$ at queue $x$, or cars, then, informally speaking, many clients remain trapped in far away slow servers, or lots of space in the road is in front of a small number of slow cars.

\subsection{Our results for convergence}

For our convergence results (from [13, 14]), we also need the following assumption on disorder. Recall that $c$ is defined by (5):

Assumption 3 We have that $c>0, \rho_{c}<+\infty$, and $\bar{R}$ satisfies the weak convexity assumption

(H) $\quad \forall \beta \in[0, c), \quad \bar{R}(\beta)-\bar{R}(c)-(\beta-c) \bar{R}^{+}(c)>0$

where $\bar{R}^{+}(c)$ is the left-hand derivative at c of the convex envelope of $\bar{R}$, that is 


$$
\bar{R}^{\prime}(c):=\limsup _{\beta \rightarrow c} \frac{\bar{R}(c)-\bar{R}(\beta)}{c-\beta} .
$$

For instance, if $R$ is strictly convex, then for any environment satisfying assumption 13,, $\bar{R}$ is strictly convex and (H) satisfied. A sufficient condition for $R$ to be strictly convex ([17]) is that $n \mapsto g(n+1)-g(n)$ is a nonincreasing function.

Theorem 1. Assume $\sqrt{13}$, Assumptions 2 and 3 . Then, for all $\eta_{0} \in \mathbb{N} \mathbb{Z}$ satisfying the supercriticality assumption

$$
\liminf _{n \rightarrow \infty} n^{-1} \sum_{x=-n}^{0} \eta_{0}(x) \geq \rho_{c}
$$

the process $\left(\eta_{t}^{\alpha}\right)_{t \geq 0}$ of initial state the configuration $\eta_{0}$ converges in distribution to $\mu_{c}^{\alpha}$ when $t \rightarrow \infty$.

Theorem 2. Assume (13), Assumptions 2 and 3 Assume further that $\eta_{0}$ satisfies

$$
\rho=\liminf _{n \rightarrow \infty} n^{-1} \sum_{x=-n}^{0} \eta_{0}(x)<\rho_{c} .
$$

Then $\eta_{t}^{\alpha}$ does not converge in distribution to $\mu_{c}^{\alpha}$ as $t \rightarrow+\infty$.

Together, as claimed, these two results give a necessary and sufficient condition for convergence to $\mu_{c}^{\alpha}$.

Now note that Theorem 1 generalises [6, Theorem 2.3] in the following ways:

1. the underlying random walk kernel is asymmetric nearest neighbour but not necessarily totally asymmetric,

2. the strict inequality in (18) is replaced by the greater than or equal condition of Theorem 1 .

3. the special case function $g(n)=\mathbf{1}_{\{n \geq 1\}}$ is removed in favour of any $g($.$) increas-$ ing to a finite limit and compatible with Assumption 3 .

In viewing these improvements, one might think that 1) could be improved to at least the condition that kernel $p($.$) is positive mean and of finite range. In fact, sur-$ prisingly, this is not possible. The nearest neighbour requirement is not to facilitate the argument. The reason is that, loosely speaking, the result holds because the incoming flux to the left of the origin is the maximal value: $c(p-q)$. This balances the outgoing flux to the right of the origin under equilibrium. How the "liminf" behaviour for the initial particle configuration is achieved is immaterial under nearest neighbour motion and for instance a configuration that is mostly vacant but which has a sparse set of very high peaks at isolated sites poses no problems. This is no longer true if the kernel is not nearest neighbour: an isolated peak with a great number of particles initially surrounded by vacant sites may be unable to furnish the needed maximal flux. The following result is established in [13]. 
Theorem 3. Assume (10) and (13). Assume further that the jump kernel $p($.$) is$ totally asymmetric (but not nearest neighbour) and $p(1)<1$. Then there exists $\eta_{0} \in \mathbb{N}^{\mathbb{Z}}$ satisfying $\left(19\right.$, such that $\eta_{t}^{\alpha}$ does not converge in distribution to $\mu_{c}^{\alpha}$ as $t \rightarrow+\infty$.

The proof of Theorem 1 comes down to showing that any limit point of the distributions $S(t) \delta_{\eta_{0}}$ must be "above" and "below" the target distribution. The upper bound almost follows from [21] which gives a strong condition for zero range processes corresponding to our conditions but with general finite range random walk kernels in $\mathbb{Z}^{d}$ for all $d \geq 1$ so that limsup $S(t) \delta_{\eta_{0}} \leq \mu_{c}^{\alpha}$. Unfortunately the following growth condition on $\eta_{0}$ is imposed in [21]:

$$
\sum_{n \in \mathbb{N}} e^{-\beta n} \sum_{x:|x|=n} \eta_{0}(x)<+\infty, \quad \forall \beta>0 .
$$

The approach of [14] is to use (for each $\varepsilon>0$ ) a comparison with finite Jackson networks on intervals $\left(A_{\varepsilon}, a_{\varepsilon}\right)$, where

$$
\begin{aligned}
A_{\varepsilon} & :=A_{\varepsilon}(\alpha)=\max \{x \leq 0: \alpha(x) \leq c+\varepsilon\}, \\
a_{\varepsilon} & :=a_{\varepsilon}(\alpha)=\inf \{x \geq 0: \alpha(x) \leq c+\varepsilon\} .
\end{aligned}
$$

These networks evolve according to the AZRP rules as if the points $A_{\varepsilon}$ and $a_{\varepsilon}$ were permanently occupied by infinitely many particles. As $\varepsilon \rightarrow 0$ the left and right endpoints tend respectively to $-\infty$ and $+\infty$. The nontrivial part was to show that this could be done so that:

(a) the resulting "finite AZRP" on $\left(A_{\varepsilon}, a_{\varepsilon}\right)$ would be positive recurrent for each $\varepsilon$;

(b) the resulting equilibria converged as $\varepsilon \rightarrow 0$ to $\mu_{c}^{\alpha}$.

The lower bound is harder to show. At root it exploits the following interface property for one-dimensional nearest neighbour processes (which will be substantially deepened in Section 5): If two configurations $\eta_{0}$ and $\xi_{0}$ satisfy the interface condition

$$
\exists x_{0}: \quad \forall y \leq x_{0}, \eta_{0}(y) \leq \xi_{0}(y) ; \forall y>x_{0}, \eta_{0}(y) \geq \xi_{0}(y)
$$

and if the AZRP's $\left(\eta_{t}\right)_{t \geq 0}$ and $\left(\xi_{t}\right)_{t \geq 0}$ are generated by the same Harris system, then the interface property is maintained:

$$
\forall t>0, \exists x_{t}: \quad \forall y \leq x_{t}, \eta_{t}(y) \leq \xi_{t}(y) ; \forall y>x_{t}, \eta_{t}(y) \geq \xi_{t}(y) .
$$

This simple property permits a recasting of the property of stochastic domination.

To show that our process $\eta_{t}^{\alpha}$ stochastically dominates $\mu_{c}^{\alpha}$ in the limit as $t$ becomes large, it is enough to show that for each $\beta<c, \eta_{t}^{\alpha}$ in the limit dominates $\mu_{\beta}^{\alpha}$; or equivalently (recalling (15)) that for each $\rho<\rho_{c}, \eta_{t}^{\alpha}$ in the limit dominates $\mu^{\alpha, \rho}$. This will be done if (for $\rho$ fixed) $\eta_{t}^{\alpha}(x) \geq \xi_{t}^{\alpha, \rho}(x)$ for $x$ fixed for $\left(\xi_{s}^{\alpha, \rho}\right)_{s \geq 0}$ an AZRP generated with the same Harris system as $\left(\eta_{s}^{\alpha}\right)_{s \geq 0}$ but initially in $\mu^{\alpha, \rho}$ equilibrium. We cannot use the interface property to compare $\eta_{t}^{\alpha}(x)$ and $\xi_{t}^{\alpha, \rho}(x)$ directly so we introduce an intermediary process $\left(\eta_{s}^{\alpha, t}\right)_{s \geq 0}$ that can be compared with both (and 
indeed everything). This process is itself generated by the same Harris system as the other two and is therefore fully defined by specifying

$$
\eta_{0}^{\alpha, t}(x)=(+\infty) \mathbf{1}_{\left\{x \leq x_{t}\right\}}
$$

where $x_{t}$ is a site that is negative, of order $t$, with environment value $\alpha\left(x_{t}\right)$ so that as $t$ becomes large, $\alpha\left(x_{t}\right)$ tends to $c$. The choice of $x_{t}$ is not straightforward, it requires assumption (H). The initial configuration (22) corresponds to placing source/sinks to the left up to site $x_{t}$ (but since jumps are nearest neighbour, as seen from the right of $x_{t}$, this is equivalent to placing a source/sink only at $x_{t}$ ).

Due to 22, there must be interfaces $I_{s}^{1}$ between $\eta_{s}^{\alpha, t}$ and $\eta_{s}^{\alpha}$ and $I_{s}^{2}$ between $\eta_{s}^{\alpha, t}$ and $\xi_{s}^{\alpha, p}$. Our domination result will follow once we have shown that with probability tending to one as $t$ becomes large

- $I_{t}^{1}$ is highly negative and

- $I_{t}^{2}$ is highly positive

as this will imply that around the origin (with high probability)

$$
\eta_{t}^{\alpha}(x) \geq \eta_{t}^{\alpha, t}(x) \geq \xi_{t}^{\alpha, \rho}(x)
$$

for all $x$ near the origin.

To show that e.g. $I_{t}^{1} \leq M_{t}$ (for some well chosen $M_{t}$ ) it suffices to show that on some interval $\left[N_{t}, M_{t}\right], \sum_{x=N_{t}}^{M_{t}}\left(\eta_{t}^{\alpha}(x)-\eta_{t}^{\alpha, t}(x)\right)>0$ (and similarly for $\left.I_{t}^{2} \geq M_{t}\right)$. We choose $N_{t}$ and $M_{t}$ to both be of order $t$ so that we can use hydrodynamic results to show that at order $t$ both $\sum_{x=N_{t}}^{M_{t}} \eta_{t}^{\alpha}(x)$ and $\sum_{x=N_{t}}^{M_{t}} \eta_{t}^{\alpha, t}(x)$ are essentially nonrandom and appropriately ordered. In fact in this calculation, it was only necessary to understand the hydrodynamic behaviour and local equilibrium starting from a source initial configuration 22).

The key point regarding this behaviour is the following. The hydrodynamic profile created by the source on its right is nonincreasing and there is critical speed $v_{c} \geq 0$ such that a front of uniform density $\rho_{c}$ propagates from the source at speed $v_{c}$. Assumption $(\mathbf{H})$ ensures that the profile is continuous at the end of this front. This enables us to choose $x_{t}$ of order $-t\left(v_{c}+\varepsilon\right)$ and ensure that 23 holds on $\left[x_{t}, 0\right]$ with any $\rho$ smaller than the hydrodynamic density created by the source around the origin, which can be made arbitrarily close to $\rho_{c}$.

A fuller picture of hydrodynamic behaviour and local equilibrium is discussed in the next sections.

\section{Hydrodynamics}

We begin with the following standard definitions in hydrodynamic limit theory. We denote by $\mathscr{M}(\mathbb{R})$ the set of Radon measures on $\mathbb{R}$. To a particle configuration $\eta \in \mathbf{X}$, we associate a sequence of empirical measures $\left(\pi^{N}(\eta): N \in \mathbb{N} \backslash\{0\}\right)$ defined by 


$$
\pi^{N}(\eta):=\frac{1}{N} \sum_{y \in \mathbb{Z}} \eta(y) \delta_{y / N} \in \mathscr{M}(\mathbb{R}) .
$$

Let $\rho_{0}(.) \in L^{\infty}(\mathbb{R})$, and let $\left(\eta_{0}^{N}\right)_{N \in \mathbb{N} \backslash\{0\}}$ denote a sequence of $\mathbf{X}$-valued random variables. We say this sequence has limiting density profile $\rho_{0}($.$) , if the sequence of$ empirical measures $\pi^{N}\left(\eta_{0}^{N}\right)$ converges in probability to the deterministic measure $\rho_{0}() d$.$x with respect to the topology of vague convergence.$

Let $f:[0,+\infty) \rightarrow \mathbb{R}$ be a Lipschitz function, and consider the conservation law

$$
\partial_{t} \rho(t, x)+\partial_{x} f[\rho(t, x)]=0 .
$$

Equation (24) means that around a point where the macroscopic particle density is $\rho$, the instantaneous algebraic flux (or current) is $f(\rho)$. This equation, with given initial condition $\rho_{0}($.$) generally does not have strong solutions even if \rho_{0}($.$) is regular,$ and has infinitely many weak solutions. However, it has a unique so called entropy solution, that is considered as the physical solution ([33]). The sequence $\left(\eta_{t}^{N}, t \geq\right.$ $0)_{N \in \mathbb{N} \backslash\{0\}}$ is said to have hydrodynamic limit $\rho(.,$.$) if: for all t \geq 0,\left(\eta_{N t}^{N}\right)_{N \in \mathbb{N} \backslash\{0\}}$ has limiting density profile $\rho(t,$.$) , which is the entropy solution to the conservation$ law (24) with initial datum $\rho_{0}($.$) .$

\subsection{Previous results for hydrodynamic limit}

The hydrodynamic limit of homogeneous asymmetric zero-range process was derived in [4, 5] for step initial conditions under the assumption of a concave flux function, and in [31] for general initial conditions without the concavity assumption (the latter result applies to more general attractive models with product invariant measures in any space dimension). In [27], the hydrodynamic behavior was studied for an AZRP with a single spatial inhomogeneity exhibiting condensation.

The paper [18] derived quenched hydrodynamics in the subcritical regime for an attractive AZRP (i.e. nondecreasing $g($.$) ), on \mathbb{Z}^{d}$, for $p($.$) finite range, with a$ disorder such that $\alpha(x)$ has a finite number of values (thus $\rho_{c}=+\infty$ ).

In the paper [32], hydrodynamics were derived through the variational coupling method, which is effective for a totally asymmetric ZRP $(p(1)=1)$, with $g(n)=$ $\mathbf{1}_{\{n \geq 1\}}$, in all regimes (subcritical, critical and supercritical).

The difficulties to prove hydrodynamic limits in our set-up are the following. At supercritical densities, there are no invariant measures; moreover we have condensation. It is thus impossible to use the traditional approach ([23]), through block averaging and block estimates, since mesoscopic block densities can blow up around condensation sites. 


\subsection{Our results on hydrodynamic limits}

The main result of [15] is the following.

Theorem 4. ([15] Theorem 2.1]) Assume the environment $\alpha$ satisfies Assumption [1] and the sequence $\left(\eta_{0}^{N}\right)_{N \in \mathbb{N} \backslash\{0\}}$ has limiting density profile $\rho_{0}(.) \in L^{\infty}(\mathbb{R})$. For each $N \in \mathbb{N} \backslash\{0\}$, let $\left(\eta_{t}^{\alpha, N}\right)_{t \geq 0}$ denote the process with initial configuration $\eta_{0}^{N}$ and generator (3). Assume either that the initial data is subcritical, that is $\rho_{0}()<.\rho_{c}^{\alpha}$; or, that Assumption 2 holds. Let $\rho(.,$.$) denote the entropy solution to (24) with initial$ datum $\rho_{0}($.$) , where f$ is the flux function defined by (25)-(26) below. Then for any $t>0$, the sequence $\left(\eta_{N t}^{\alpha, N}\right)_{N \in \mathbb{N} \backslash\{0\}}$ has limiting density profile $\rho(t,$.$) .$

To complete the above theorem, we explain how the flux function in 24 is obtained. Let $x$ : $t \mapsto x_{t}$ be a $\mathbb{Z}$-valued path representing the position of a moving "observer" on the lattice. We let $\Gamma_{x,}(t, \eta)$ denote the algebraic current across $x$, that is the algebraic number of particles crossing the "observer" to the right, between times 0 and $t$, when starting from the initial configuration $\eta$. In the special case where $x$. is identically 0 , we obtain the current through the origin between times 0 and $t$, simply denoted by $\Gamma_{0}^{\alpha}(t, \eta)$ : that is, the number of jumps from 0 to 1 minus the number of jumps from 1 to 0 . Then the flux function $\rho \mapsto f(\rho)$ is defined by

$$
f(\rho):=\lim _{t \rightarrow+\infty} t^{-1} \Gamma_{0}^{\alpha}\left(t, \eta^{\rho}\right) .
$$

We can show that this limit exists and depends only on $\rho$ (and not on the choice of $\eta^{\rho}$ nor on $\alpha$ ), where $\eta^{\rho}$ denotes a configuration with density $\rho$, that is a configuration satisfying 16 . For $\beta \in[0, c)$, we can compute the stationary current under $\mu_{\beta}^{\alpha}$, as follows.

$$
\int_{\mathbf{X}}[p \alpha(x) g(\eta(x))-q \alpha(x+1) g(\eta(x+1))] d \mu_{\beta}^{\alpha}(\eta)=(p-q) \beta .
$$

Recall the function $\bar{R}$ defined by $[13$. As a function of the mean density $\rho=\bar{R}(\beta)$, we define the flux for our system as

$$
f(\rho):=(p-q) \bar{R}^{-1}(\rho) .
$$

And we extend $f$ to $\left[\rho_{c},+\infty\right)$ by

$$
f(\rho)=(p-q) c, \quad \forall \rho \geq \rho_{c} .
$$

Remark 3. Under Assumption (EE) of an ergodic environment, the flux function depends only on the marginal $Q_{0}$ of the environment. Under the more general Assumptions 1 , 2 , the flux function depends on the pair $\left(Q_{0}, c\right)$ defined in (11)- 13$)$. In the latter case, the inequality in (11) can be strict, so $c$ should be regarded as an additional parameter not contained in $Q_{0}$, whereas in the ergodic case, equality always holds in (11). A simple non-ergodic example is given in Subsection 5.3 below. 
To prove Theorem 4, we use a reduction principle established in [7, 8, 9, 10, 11] for one dimensional conservative attractive processes. This method reduces the proof of hydrodynamics for a Cauchy initial condition to that for a Riemann initial condition, that is, of the form

$$
\rho_{0}(x)=R_{\lambda, \rho}(x):=\lambda \mathbf{1}_{\{x<0\}}+\rho \mathbf{1}_{\{x \geq 0\}}
$$

for $\lambda, \rho \in \mathbb{R}$. The passage from one to the other is similar in spirit to Riemannbased numerical schemes for scalar conservation laws; the difficulty is to control the propagation of the error committed at successive time steps when replacing the actual entropy solution with a piecewise constant approximation. Crucial tools in this reduction are:

(i) The finite propagation property, that is the fact that discrepancies between two AZRP's, and similarly between two entropy solutions of [24, propagate with bounded speed.

(ii) The macroscopic stability property, which states that if two AZRP configurations are initially close macroscopically, they remain so at later times. In our case this is a consequence of the fact that jumps are nearest neighbour and $g($. is nondecreasing.

To prove hydrodynamics for the Riemann initial condition (27), we use a variational characterization (see e.g. [11]) of the entropy solution $R_{\lambda, \rho}(t,$.$) of the Riemann$ problem (that is, 24) with initial datum (27)). Namely, define

$$
\mathscr{G}_{\lambda, \rho}(v)=\left\{\begin{array}{l}
\min _{r \in[\lambda, \rho]}[f(r)-v r] \text { if } \lambda \leq \rho, \\
\max _{r \in[\rho, \lambda]}[f(r)-v r] \text { if } \lambda \geq \rho .
\end{array}\right.
$$

For such values, $R_{\lambda, \rho}(t, v t)$ is the minimizer (resp. maximizer) in 28$)$ if $\lambda \leq \rho$ (resp. $\lambda \geq \rho$ ). This optimizer is unique for all but countably many values of $v$.

In order to prove 28 at the microscopic level, the main issue is to show that

$$
\lim _{t \rightarrow+\infty} t^{-1} \Gamma_{x}\left(t, \xi^{\alpha, \lambda, \rho}\right)=\mathscr{G}_{\lambda, \rho}(v)
$$

in probability, where $x$. is a path with asymptotic speed $v$, and $\xi^{\alpha, \lambda, \rho}$ is a random configuration with profile $R_{\lambda, \rho}($.$) in 27$. Assume for instance $\lambda<\rho$. To define a suitable configuration $\xi^{\alpha, \lambda, \rho}$ in 29, we use a family of AZRP's $\xi^{\alpha, r, r}$, where $r \geq 0$, and $\xi^{\alpha, r, r}$ has homogeneous macroscopic density $r$ at time 0 . For $r<\rho_{c}$, we can choose $\xi^{\alpha, r, r}$ to be an equilibrium process with density $r$ (that is, with distribution $\mu^{\alpha, r}$, cf. (15)). For $r>\rho_{c}$, such equilibria do not exist. Instead, we use what we call "pseudo-equilibria", that is, configurations $\xi^{\alpha, r, r}$ with a supercritical homogeneous macroscopic density profile. We choose $\xi^{\alpha, \lambda, \rho}$ in 29, as the configuration whose restriction to $x \leq 0$ is $\xi^{\alpha, \lambda, \lambda}$ and whose restriction to $x>0$ is $\xi^{\alpha, \rho, \rho}$. The following main ideas are then involved to derive 29): 
(a) We prove convergence 29 for equilibria and pseudo-equilibria, that is $\lambda=$ $\rho=r$. This follows from ergodicity in the case $r<\rho_{c}$ of equilibria, but novel arguments are necessary in the case $r>\rho_{c}$ of pseudo-equilibria.

(b) The upper bound in 29 (proving that the 1.h.s. is dominated with high probability by the r.h.s.) is the simpler part. It follows from a coupling argument showing that the current in $\xi^{\alpha, \lambda, \rho}$ cannot exceed the one in $\xi^{\alpha, r, r}$. This property can be regarded as "intuitive" because, since $r \in[\lambda, \rho]$, the latter system has initially more particles to the left and more space to the right. However, mathematically, this is related to the macroscopic stability property.

(c) For the lower bound in 29, we introduce a novel "interface process", which gives a more adapted (in our setting) version of the local particle density than the usual block average. This is a random spatially nondecreasing lattice field $\rho_{t}(x)$ taking values in $[\lambda, \rho]$ with the following property: in a space region where $\rho_{t}$ does not fluctuate much, the system is approximately at local equilibrium or pseudo-equilibrium, in the sense that $\xi_{t}^{\alpha, \lambda, \rho}$ is close to $\xi_{t}^{\alpha, r, r}$ for a random $r=$ $\rho_{t}(x)$. As a nondecreasing function cannot jump too often, for "most" values of $v$, using $(a)$, the macroscopic current across a path with velocity $v$ is close to $f\left[\rho_{t}(v t)\right]-v \rho_{t}(v t)$, which dominates the minimum in 28). Whence the desired lower bound.

The interface process is obtained by looking at simultaneous interfaces of $\xi^{\alpha, \lambda, \rho}$ in the sense of 201-21 with all equilibria and pseudo-equilibria $\xi^{\alpha, r, r}$ for $r \in$ $[\lambda, \rho]$. Precisely, we can define a simultaneous evolution of interfaces $x^{r}$. between $\eta^{\alpha}$. and $\xi^{\alpha, r, r}$ so that $x_{t}^{r}$ is nondecreasing with respect to $r$, and define $x \mapsto \rho_{t}(x)$ as a generalized inverse of $r \mapsto x_{t}^{r}$.

\subsection{Examples}

In this subsection, we illustrate the behaviour of the flux function and of solutions to 24] with some examples.

Dilute limit. It is natural to compare the "disordered" flux function $25-26$ to the "homogeneous" flux function $f_{\text {hom }}$ obtained from the same AZRP in a homogeneous environment $\alpha_{\mathrm{hom}}(x) \equiv 1$. In general, there is no simple relation between these two fluxes. However, the relation becomes simple and natural in the so-called dilute limit where $\alpha(x)=1$ at "most" sites. More precisely, let $Q_{0}$ be the probability measure in Assumption 1. (EE) and (17). We consider an i.i.d. random environment in which, for each $x \in \mathbb{Z}, \alpha(x)$ is chosen chosen according to $Q_{0}$ with probability $\varepsilon \geq 0$. and equal to 1 with probability $(1-\varepsilon)$. Thus $\alpha(x)$ has distribution

$$
Q_{0}^{\varepsilon}:=(1-\varepsilon) \delta_{1}+\varepsilon Q_{0} .
$$

The value $\varepsilon=0$ corresponds to the homogeneous environment $\alpha_{\text {hom }}$, while $\varepsilon=1$ corresponds to the i.i.d. environment with marginal $Q_{0}$. The dilute limit is the limit 
$\varepsilon \rightarrow 0+$. Let $f^{\varepsilon}$ denote the flux function $25-26$ produced by the environment with marginal 30 . For $\varepsilon=0, f^{0}=f_{\text {hom }}$ is the flux function for the homogenous AZRP. It follows from $25-26$, that

$$
f_{\text {hom }}(\rho)=(p-q) R^{-1}(\rho) .
$$

For $\varepsilon \in(0,1]$, there is no simple relation between $f^{\varepsilon}$ and $f_{\text {hom }}$. However, using (25) $-(26)$ and (17), we can show that

$$
\lim _{\varepsilon \rightarrow 0} f^{\varepsilon}(\rho)=f_{d}(\rho)
$$

where $f_{d}$ is the dilute limit of the flux function, defined by

$$
f_{d}(\rho):=\left\{\begin{array}{l}
f_{\text {hom }}(\rho) \text { if } \rho<\rho_{c} \\
(p-q) c \text { if } \rho \geq \rho_{c}
\end{array}\right\}=f_{\text {hom }}(\rho) \wedge(p-q) c .
$$

This limit can be understood intuitively as follows. As $\varepsilon \rightarrow 0$, slow sites are very rare, hence the system exhibits long homogeneous stretches where it behaves as a homogeneous process. Thus the memory of slow sites is only retained by the flux truncation, but not by the shape of the flux function prior to truncation. Note in particular that only the infimum of the support of $Q_{0}$ in 30 ) (but not details of the distribution) is involved in the dilute limit.

We defined above the dilute limit from the limit of a sequence of random environments. Another point of view is to construct a single deterministic environment equal to 1 except on a 0 density subset of $\mathbb{Z}$, where it asymptotically approaches its infimum value $c$. Precisely, let $\alpha($.$) be an environment satisfying the conditions of$ Assumption 2, with values in $[c, 1]$, and such that

$$
\alpha(x)=1 \text { if } x \notin\left\{x_{n}: n \in \mathbb{Z}\right\} .
$$

Assume moreover $\lim _{n \rightarrow \pm \infty}\left(n / x_{n}\right)=0$. For such an environment, the flux function defined by 25]-26) is exactly $f_{d}$ given by (32). As announced in Remark 3 , this example shows that, outside the case of an ergodic random environment, the flux function is not entirely determined by the empirical distribution $Q_{0}$ in 9 . Indeed, in this case we have $Q_{0}=\delta_{1}$, which does not give any information on $c$.

Supercritical entropy solutions. We now describe the consequences of the flat line 26] on the behaviour of entropy solutions through the analysis of the so-called Riemann problem with initial data of the form (27). The following result can be obtained (see [15]) using [28].

Proposition 1. Assume $+\infty>\lambda \geq \rho_{c}>\rho$. Let

$$
v_{c}(\rho):=\inf _{r \in\left[\rho, \rho_{c}\right)} \frac{f\left(\rho_{c}\right)-f(r)}{\rho_{c}-r}=\inf _{r \in\left[\rho, \rho_{c}\right)} \frac{\widehat{f}\left(\rho_{c}\right)-\widehat{f}(r)}{\rho_{c}-r}=\widehat{f}^{\prime}\left(\rho_{c}-\right)
$$

where $\widehat{f}$ denotes the concave envelope of $f$ on $\left[\rho, \rho_{c}\right]$. In particular, if $f$ is concave, 


$$
v_{c}(\rho)=f^{\prime}\left(\rho_{c}-\right)=\left\{\int_{[c, 1]} \frac{1}{a} R^{\prime}\left[\frac{c}{a}\right] d Q_{0}(a)\right\}^{-1} .
$$

Then, for every $t>0$, we have

$$
\begin{aligned}
R_{\lambda, \rho}(x, t) & =\lambda, \quad \forall x<0 \\
R_{\lambda, \rho}(x, t) & =R_{\rho_{c}, \rho}(x, t), \quad \forall x>0 \\
\lim _{t \rightarrow+\infty} R_{\lambda, \rho}(x, t) & =\rho_{c}, \quad \forall x \geq 0 \\
R_{\lambda, \rho}(0+, t) & =\rho_{c} \\
R_{\lambda, \rho}(x, t) & =\rho_{c}, \quad \forall x \in\left(0, t v_{c}(\rho)\right) \\
R_{\lambda, \rho}(x, t) & <\rho_{c}, \quad \forall x>t v_{c}(\rho)
\end{aligned}
$$

Property (34) states that the initial constant density is not modified to the left of the origin. This is related to the fact that $f$ is nondecreasing, hence characteristic velocities are always nonnegative.

Property (35) states that the solution to the right of the origin does not depend on the supercritical initial density $\lambda$ on the left. Formally, we may thus consider that this is also the solution for " $\lambda=+\infty$ ", which corresponds to placing sources to the left of the origin. In particular, for $\rho=0$, we recover the source solution used in Subsection 4.2, that is the hydrodynamic limit starting from the particular source configuration (22).

Properties 36, 37, 38 are signatures of the phase transition. They express the fact that, regardless of the supercritical value on the left side, supercritical densities are blocked, and the right side is dominated by the critical density. In particular, (38)-(39) state that a front of critical density propagates to the right from the origin at speed $v_{c}$ if $v_{c}(\rho)>0$.

An explicit case: $M / M / 1$ queues in series. Consider the $M / M / 1$ queues in series, that is $g(n)=\min (n, 1)$, with total asymmetry $(p=1, q=0)$, cf. Sect. 2. With this choice of $g,(12)$ and 31 write

$$
R(\beta)=\frac{\beta}{1-\beta}, \quad f_{\mathrm{hom}}(\rho)=\frac{\rho}{1+\rho} .
$$

In view of (40), the dilute limit $f_{d}$ defined in (32) writes here

$$
f_{d}(\rho)=\left[\frac{\rho}{1+\rho}\right] \wedge c= \begin{cases}\frac{\rho}{1+\rho} & \text { if } \rho<\rho_{c}:=\frac{c}{1-c} \\ c & \text { if } \rho \geq \rho_{c} .\end{cases}
$$

Since $f_{d}$ defined by (41) is concave, (33) yields

$$
v_{c}(\rho)=f_{\text {hom }}^{\prime}\left(\rho_{c}^{-}\right)=(1-c)^{2} .
$$




\section{Local equilibrium}

We now come to results on strong local equilibrium with respect to the hydrodynamic limit (24).

A natural extension of the convergence theorems is to establish results for the following question. Let us fix a realization of the environment $\alpha($.$) and suppose$ given a sequence of initial configurations $\left\{\eta_{0}^{N}\right\}_{N \geq 0}$ which correspond to a "profile" $\rho_{0}$ in the sense that, for every $a, b \in \mathbb{R}$,

$$
\frac{1}{N} \sum_{a N<k<b N} \eta_{0}^{N}(k)-\int_{a}^{b} \rho_{0}(x) d x \rightarrow 0 .
$$

Let $x_{N} \in \mathbb{Z}$ satisfy $N^{-1} x_{N} \rightarrow u \in(-\infty, \infty)$. What can be said about the behaviour of $\eta_{N t}^{\alpha, N}$ around $x_{N}$ ?

We suppose that the entropy solution $\rho(.,$.$) to the associated hydrodynamic equa-$ tion 24 is continuous at $(t, u)$. It is reasonable to believe that $\tau_{\left[x_{N}\right]} \eta_{N t}^{\alpha, N}$ "looks" like the equilibrium corresponding to $\rho(t, u)$ (with environment $\alpha$ suitably shifted). This question is anticipated by several works on conservative systems without disorder. In translation invariant cases where there is a family of equilibria $\left\{\mu^{r}\right\}_{r \geq 0}$ and the initial configurations are random there is conservation of local equilibrium if, when

$$
\lim _{N \rightarrow \infty} \tau_{[u N]} \eta_{0}^{N}=\mu^{\rho_{0}(u)}
$$

in distribution for each continuity point $u$ of $\rho_{0}($.$) and for each (t, u)$ as above, then

$$
\lim _{N \rightarrow \infty} \tau_{[u N]} \eta_{N t}^{N}=\mu^{\rho(t, u)}
$$

in distribution. In [26], conservation of local equilibrium was proved for a homogeneous zero-range process with a strictly convex flux, under an initial invariant product measure. Following [2], [12] showed for finite range nonzero mean exclusion processes, that 43 held with no further assumptions on $\eta_{0}^{N}$ beyond the profile hypothesis (42). We call this a "spontaneous creation of local equilibrium."

In our family of models it is natural to hope for similar results. We discuss three differences:

1. Our system is not translation invariant and in fact we cannot expect a limit as in 43 because the environment varies as $N \rightarrow+\infty$ : given appropriate $(t, u)$ it is possible to find sequences $x_{N}$ and $y_{N}$ that converge macroscopically to $x$ so that $\mathbf{E}\left(f\left(\tau_{x_{N}} \eta_{N t}^{\alpha, N}\right)\right)-\mathbf{E}\left(f\left(\tau_{y_{N}} \eta_{N t}^{\alpha, N}\right)\right)$ does not converge to zero as $N$ becomes large.

2. Around a fixed point, say 0 , the environment is fixed and will satisfy $\alpha(x)<c$ for $x$ close to the point, however when considering $x_{N}$ which (in scale $N$ ) converges to, say the origin, it is perfectly possible that $\alpha\left(x_{N}\right)$ converges to $c$.

3 . The value $\rho(t, u)$ may be supercritical, that is strictly greater than $\rho_{c}$ the maximum density for an equilibrium. 
The first point is dealt with by a simple reformulation of the result but the second and third questions require a more substantive response. The second requires us to consider AZRP for which the occupation number $+\infty$ is permitted. For the third, as with other papers analyzing condensation phenomena, we expect that the limiting density will be $\rho_{c}$ and not $\rho(t, u)$.

Our first results concern cases where the entropy solution $\rho(.,$.$) is continuous at$ $(t, u)$ and has a value strictly below $\rho_{c}$.

Theorem 5. Under assumptions of Theorem 4 the following holds for every $(t, u) \in$ $(0,+\infty) \times \mathbb{R}$ : let $\psi: \mathbf{X} \rightarrow \mathbb{R}$ be a bounded local function, and $\left(x_{N}\right)_{N \in \mathbb{N}}$ a sequence of sites such that $u=\lim _{N \rightarrow+\infty} N^{-1} x_{N}$. Then if $\rho(.,$.$) is continuous at (t, u)$ and $\rho(t, u)<\rho_{c}$,

$$
\lim _{N \rightarrow+\infty}\left[\mathbf{E} \psi\left(\tau_{x_{N}} \eta_{N t}^{\alpha, N}\right)-\int_{\mathbf{X}} \psi(\eta) d \mu^{\tau_{\tau_{N}} \alpha, \rho(t, u)}(\eta)\right]=0 .
$$

This result relied upon coupling. It is sufficient to show the desired convergence for increasing cylinder functions $\psi$. So it is sufficient to show that for $r<\rho(t, u)$ we have

$$
\liminf _{N \rightarrow \infty} \mathbf{E} \psi\left(\tau_{x_{N}} \eta_{N t}^{\alpha, N}\right)-\int_{\mathbf{X}} \psi(\eta) d \mu^{\tau_{x_{N}} \alpha, r}(\eta) \geq 0
$$

for $N$ large; and similarly for $r>\rho(t, u)$. So it will be sufficient to show that for $N$ large in a $N$ order interval centered around $x_{N}$, the process $\eta_{t N}^{\alpha, N}$ dominates $\xi_{N t}^{\alpha, r}$ where as before $\xi_{. t}^{\alpha, r}$ is a AZRP run with the given Harris system in $\mu^{\alpha, r}$ equilibrium.

Our approach shadows that of [12] but is a bit more complicated than this argument which dealt with the exclusion process. In particular the key tool of [24], which relies on global strict concavity or convexity of the flux function, is no longer available here.

Under the general assumptions on function $g($.$) , the flux function f$ need not be globally convex or globally concave. Nonetheless away from the critical value the function $f$ is analytic and therefore in any interval of densities, there will exist subintervals on which the flux is either globally convex or globally convex. Thus given $r<\rho(t, u)$ we can find $r<r_{1}<r_{2}<\rho(t, u)$ so that $f$ is either convex or concave on $\left(r_{1}, r_{2}\right)$. For concreteness suppose it is concave, then we may "impose" a system of priorities (or classes, cf. [3]) on $\eta_{s}^{\alpha, N}$ particles that are not coalesced with $\xi_{s}^{\alpha, r}$ particles. This means that higher priority particles will be faster than lower priority ones. So a given small, but order $N$, interval $I$ can be placed between two similar intervals $J_{l}$ and $J_{r}$. In a short time (unless they become coalesced) fast particles in $J_{l}$ will overtake slow particles in $J_{r}$. This entails coalescence of many uncoalesced $\xi_{s}^{\alpha, r}$ particles originally in $I$. This argument is repeated until in an interval around $x_{N}$, the "density" of uncoalesced $\xi_{s}^{\alpha, r}$ particles will be very small. We can then argue as in [12] to show the required domination.

Next, we consider points at which the hydrodynamic density is supercritical or critical. As discussed above, there does not exist a corresponding equilibrium mea- 
sure, so one cannot expect the same type of convergence as above. We expect and to some extent show one has local convergence to the critical quenched invariant measure. We now introduce an additional assumption that is needed.

Definition 1. Let $\left(x_{N}\right)_{N \in \mathbb{N}}$ be a sequence of sites such that $N^{-1} x_{N}$ converges to $u \in$ $\mathbb{R}$ as $N \rightarrow+\infty$. The sequence $\left(x_{N}\right)_{N \in \mathbb{N}}$ is typical if and only if any subsequential limit $\bar{\alpha}$ of $\left(\tau_{x_{N}} \alpha\right)_{N \in \mathbb{N}}$ has the following properties:

(i) For every $z \in \mathbb{Z}, \bar{\alpha} \in \mathbf{B}:=(c, 1]^{\mathbb{Z}}$.

(ii) $\liminf _{z \rightarrow-\infty} \bar{\alpha}(z)=c$.

The interpretation of the word "typical" is the following. When $\alpha$ does not achieve its infimum value $c$ (that is $\alpha \in \mathbf{B}$ ), (i)-(ii) says that the environment as seen from $x_{N}$ shares key properties of the environment seen from a fixed site (say the origin).

The typicality assumption will in fact be needed only to show that the microscopic distribution is locally dominated by the critical measure. We observe however that even in the critical case, where the invariant measure does exist, we are not able to prove the statement without the typicality assumption.

Theorem 6. Under assumptions of Theorem 4 the following holds for every $(t, u) \in$ $(0,+\infty) \times \mathbb{R}$. Let $\psi: \mathbf{X} \rightarrow \mathbb{R}$ be a bounded local function, and $\left(x_{N}\right)_{N \in \mathbb{N}}$ a typical sequence of sites such that $u=\lim _{N \rightarrow+\infty} N^{-1} x_{N}$. Then:

(i) If $\psi$ is nondecreasing,

$$
\lim _{N \rightarrow+\infty}\left[\mathbf{E} \psi\left(\tau_{x_{N}} \eta_{N t}^{\alpha, N}\right)-\int_{\overline{\mathbf{x}}} \psi(\eta) d \mu_{c}^{\tau_{x_{N}} \alpha}(\eta)\right]^{+}=0 .
$$

(ii) If $\rho_{*}(t, u) \geq \rho_{c}$ (where $\left.\rho_{*}(t, u):=\liminf _{\left(t^{\prime}, u^{\prime}\right) \rightarrow(t, u)} \rho\left(t^{\prime}, u^{\prime}\right)\right)$, then

$$
\lim _{N \rightarrow+\infty}\left[\mathbf{E} \psi\left(\tau_{x_{N}} \eta_{N t}^{\alpha, N}\right)-\int_{\overline{\mathbf{x}}} \psi(\eta) d \mu_{c}^{\tau_{x_{N}} \alpha}(\eta)\right]=0
$$

The argument we give for the upper bound is now close to that for the convergence upper bound: we show that we can find $y_{N}<x_{N}<z_{N}$ so that the "finite" AZRP on $\left(y_{N}, z_{N}\right)$ with infinitely many particles at $y_{N}$ and $z_{N}$ has an equilibrium very close to $\mu_{c}^{\alpha}$. The lower bound is essentially supplied by the arguments for Theorem 5

Without the above typicality assumption, we are not able to prove such a statement at given times, but we can obtain a weaker time-integrated result.

Theorem 7. Under assumptions and notations of Theorem 4 the following holds. Let $t>0$, and $\left(x_{N}\right)_{N \in \mathbb{N}}$ be an arbitrary sequence of sites such that $\lim _{N \rightarrow+\infty} N^{-1} x_{N}=$ $u$, where $u \in \mathbb{R}$ is such that $\rho_{*}(t, u) \geq \rho_{c}$. Let $\psi: \overline{\mathbf{X}} \rightarrow \mathbb{R}$ be a continuous local function. Then

$$
\lim _{\delta \rightarrow 0} \limsup _{N \rightarrow+\infty}\left|\frac{1}{\delta} \int_{t-\delta}^{t} \mathbf{E} \psi\left(\tau_{x_{N}} \eta_{N s}^{\alpha, N}\right) d s-\int_{\overline{\mathbf{X}}} \psi(\eta) d \mu_{c}^{\tau_{x_{N}} \alpha}(\eta)\right|=0 .
$$


Remark 4. Whereas in Theorems 5 and 6, the test function $\psi$ is defined on $\mathbf{X}$, in Theorem 7 , it is defined on $\overline{\mathbf{X}}$. Indeed in Theorem 5 , the fact that $\rho(t, u)<\rho_{c}$ implies that the subcritical measure $\mu^{\tau_{x_{N}} \alpha, \rho(t, u)}$ in 44 is supported on $\mathbf{X}$. In Theorem 6 , statement $(i)$ in Definition 11 of the typicality assumption ensures that the critical measure $\mu_{c}^{\tau_{x_{N}} \alpha}$ in 45- 46 is still supported on $\mathbf{X}$. However in Theorem 7, owing to the absence of the typicality assumption, the critical measure $\mu_{c}^{\tau_{x_{N}} \alpha}$ in 47 may have infinitely many particles at sites $x \in \mathbb{Z}$ such that $\alpha(x)=c$.

Our approach is to first note that, again, the lower bound is achieved via Theorem 5. The upper bound is more intricate. We argue that in the supercritical regime the current through any point must ("on average") equal $c$. We then argue that any invariant measure that dominates $\mu_{c}^{\alpha}$ and has current $c$ must equal $\mu_{c}^{\alpha}$. The (unfortunately necessary) Cesàro means are to give an invariant measure for any limit measure and to ensure that the limit measure must have flux $c$.

Example. An illuminating particular case of the above theorems is when the initial datum is uniform. Assume

$$
\rho_{0}(x) \equiv \rho
$$

for some $\rho \geq 0$. Then $\rho(t, x) \equiv \rho$ for all $t>0$. Specializing Theorems 5 and 6 to $u=0$, we obtain that $\eta_{N t}^{\alpha, N}$ converges in distribution to $\mu^{\alpha, \rho}$ if $\rho<\rho_{c}$, or to $\mu_{c}^{\alpha}$ if $\rho \geq \rho_{c}$. We may in particular achieve (48) as follows by a sequence of initial configurations $\eta_{0}^{N}=\eta_{0}$ independent of $N$.

(i) Stationary initial state. Let $\eta_{0}^{N}=\eta_{0} \sim \mu^{\alpha, \rho}$, with $\rho<\rho_{c}$. Since $\mu^{\alpha, \rho}$ is an invariant measure for the process with generator (3), for every $t>0$, we have $\eta_{N t}^{\alpha, N} \sim \mu^{\alpha, \rho}$. As a result, the expression between brackets in 44 vanishes for every $N \in \mathbb{N} \backslash\{0\}$ and $t \geq 0$. Hence there is conservation of local equilibrium (since (44) already holds for $t=0$ ), but there is in fact nothing to prove, since this conservation follows form stationarity.

(ii) Deterministic initial state. Let $\eta_{0}^{N}=\eta_{0}$, where $\eta_{0} \in \mathbf{X}$ has density $\rho$, cf. 16. This implies that the sequence $\left(\eta_{0}^{N}\right)_{N \in \mathbb{N} \backslash\{0\}}$ has density profile $\rho_{0}(x) \equiv \rho$. Here Theorems 5 and 6 are no longer void statements as in (i). When specialized to $u=$ 0 , they yield a large-time convergence result for our process. Namely, Theorem 5 implies that $\eta_{t}^{\alpha} \rightarrow \mu^{\alpha, \rho}$ in distribution if $\rho<\rho_{c}$, and Theorem6 6implies $\eta_{t}^{\alpha} \rightarrow$ $\mu_{c}^{\alpha}$ if $\rho \geq \rho_{c}$.

Pushing the analysis of (ii) further, we can show that the first limit in (16) is irrelevant, and derive from Theorems 5 and 6 the following convergence result.

Theorem 8. Let $\eta_{0} \in \mathbf{X}$ be such that, for some $\rho>0$,

$$
\lim _{n \rightarrow+\infty} \frac{1}{n} \sum_{x=-n}^{0} \eta_{0}(x)=\rho
$$

Then $\eta_{t}^{\alpha}$ converges in distribution as $t \rightarrow+\infty$ to $\mu^{\alpha, \rho \wedge \rho_{c}}$. 
Remark 5. (i) In the case $\rho<\rho_{c}$, Theorem 8 solves the convergence problem posed in [18, pp 195-196].

(ii) In the case $\rho \geq \rho_{c}$, Theorem 8 is a partial improvement over Theorem 1 It improves the latter in the sense that we do not need a weak convexity assumption on $g$, but is it less general with respect to initial conditions.

Acknowledgements C.B. and E.S. thank respectively the organizers of PSPDE V and PSPDE VII for their invitation to participate in these conferences, and therefore giving them the opportunity to present their work, then to write the present text.

This work was partially supported by laboratoire MAP5, grants ANR-15-CE40-0020-02 and ANR-14-CE25-0011, Simons Foundation Collaboration grant 281207 awarded to K. Ravishankar. This work has been conducted within the FP2M federation (CNRS FR 2036). C.B., T.M. and K.R. thank Université Paris Descartes for hospitality.

\section{References}

1. Andjel, E.D.: Invariant measures for the zero-range process. Ann. Probab. 10, 525-547 (1982)

2. Andjel, E.D.: The asymmetric simple exclusion process on $\mathbb{Z}^{d}$. Z. Wahrscheinlichkeitstheor. Verw. Geb. 58, 423-432 (1981)

3. Andjel, E.D., Kipnis, C.: Derivation of the hydrodynamical equation for the zero-range interaction process. Ann. Probab.12, no. 2, 325-334 (1984)

4. Andjel, E.D., Vares, M.E.: Hydrodynamic equations for attractive particle systems on $\mathbb{Z}$. J. Stat. Phys. 47, no. 1-2, 265-288 (1987)

5. Andjel, E.D., Vares, M.E.: Correction to: Hydrodynamic equations for attractive particle systems on $\mathbb{Z}$. J. Stat. Phys. 113, no. 1-2, 379-380 (2003)

6. Andjel, E., Ferrari, P.A., Guiol, H., Landim, C.: Convergence to the maximal invariant measure for a zero-range process with random rates. Stoch. Proces. Appl. 90, 67-81 (2000)

7. Bahadoran, C., Guiol, H., Ravishankar, K., Saada, E.: A constructive approach to Euler hydrodynamics for attractive particle systems. Application to $k$-step exclusion. Stoch. Process. Appl. 99, no. 1, 1-30 (2002)

8. Bahadoran, C., Guiol, H., Ravishankar, K., Saada, E.: Euler hydrodynamics of onedimensional attractive particle systems. Ann. Probab. 34, no. 4, 1339-1369 (2006)

9. Bahadoran, C., Guiol, H., Ravishankar, K., Saada, E.: Strong hydrodynamic limit for attractive particle systems on $\mathbb{Z}$. Elect. J. Probab. 15, no. 1, 1-43 (2010)

10. Bahadoran, C., Guiol, H., Ravishankar, K., Saada, E.: Euler hydrodynamics for attractive particle systems in random environment. Ann. Inst. H. Poincaré Probab. Statist. 50, no. 2 , 403-424 (2014)

11. Bahadoran, C., Guiol, H., Ravishankar, K., Saada, E.: Constructive Euler hydrodynamics for one-dimensional attractive particle systems. in: Sojourns in Probability Theory and Statistical Physics - III - Interacting Particle Systems and Random Walks, A Festschrift for Charles M. Newman., 43-89; Sidoravicius, V. (ed.); Springer PROMS, Vol. 300, Springer, Singapore (2019)

12. Bahadoran, C., Mountford, T. S.: Convergence and local equilibrium for the one-dimensional nonzero mean exclusion process. Probab. Theory Related Fields 136, no. 3, 341-362 (2006)

13. Bahadoran, C., Mountford, T.S., Ravishankar, K., Saada, E.: Supercriticality conditions for the asymmetric zero-range process with sitewise disorder. Braz. J. Probab. Stat. 29, no. 2 , 313-335 (2015)

14. Bahadoran, C., Mountford, T.S., Ravishankar, K., Saada, E.: Supercritical behavior of zerorange process with sitewise disorder. Ann. Inst. H. Poincaré Probab. Statist. 53, no. 2, 766-801 (2017) 
15. Bahadoran, C., Mountford, T.S., Ravishankar, K., Saada, E.: Hydrodynamics in a condensation regime: the disordered asymmetric zero-range process. Ann. Probab. 48, no. 1, 404-444 (2020)

16. Bahadoran, C., Mountford, T.S., Ravishankar, K., Saada, E.: Quenched convergence and strong local equilibrium for asymmetric zero-range process with sitewise disorder. Probab. Theory Rel. Fields, 176, no. 1-2, 149-202 (2020)

17. Balázs, M., Seppäläinen T.: A convexity property of expectations under exponential weights. Arxiv: PR07074273 (2007).

18. Benjamini, I., Ferrari, P.A., Landim, C.: Asymmetric conservative processes with random rates. Stochastic Process. Appl. 61, no. 2, 181-204 (1996)

19. Evans, M.R.: Bose-Einstein Condensation in Disordered Exclusion Models and Relation to Traffic Flow. Europhysics Letters, 36, no. 1, 13 (1996)

20. Ferrari, P., Krug, J.: Phase transitions in driven diffusive systems with random rates. J. Phys. A. 29, L:465-471 (1996)

21. Ferrari, P.A., Sisko, V.V.: Escape of mass in zero-range processes with random rates. in: Asymptotics: particles, processes and inverse problems, 108-120, IMS Lecture Notes Monogr. Ser., 55, Inst. Math. Statist., Beachwood, OH (2007)

22. Harris, T.E.: Nearest-neighbour Markov interaction processes on multidimensional lattices. Adv. Math 9, 66-89 (1972)

23. Kipnis, C., Landim, C.: Scaling limits for interacting particle systems. Springer (1999)

24. Kosygina E.: The behaviour of specific entropy in the hydrodynamic scaling limit. Ann. Probab. 29, no. 3, 1086-1110 (2001)

25. Krug, J.: Phase separation in disordered exclusion models Brazilian Journal of Physics, 30, 97-104 (2000)

26. Landim, C.: Conservation of local equilibrium for attractive particle systems on $\mathbb{Z}$. Ann. Probab. 21, no. 4, 1782-1808 (1993)

27. Landim, C.: Hydrodynamical limit for space inhomogeneous one-dimensional totally asymmetric zero-range processes. Ann. Probab. 24, 599-638 (1996)

28. Liggett, T.M.: Interacting particle systems. Reprint of the 1985 original. Classics in Mathematics. Springer-Verlag, Berlin (2005)

29. Mountford, T.: An extension of a result of Andjel. Ann. Applied Probab. 11, 321-337 (2001)

30. Pardoux, E.: Processus de Markov et applications. Dunod, Paris (2007)

31. Rezakhanlou, F.: Hydrodynamic limit for attractive particle systems on $\mathbb{Z}$. Comm. Math. Phys. 140, no. 3, 417-448 (1991)

32. Seppäläinen, T., Krug, J.: Hydrodynamics and platoon formation for a totally asymmetric exclusion process with particlewise disorder. J. Stat. Phys. 95, 525-567 (1999)

33. Serre, D.: Systems of conservation laws. 1. Hyperbolicity, entropies, shock waves. Translated from the 1996 French original by I.N. Sneddon. Cambridge University Press, Cambridge (1999) 